\title{
Novel amidases of two Aminobacter sp. strains: Biotransformation experiments and elucidation of gene sequences
}

\author{
Ulrike Engel", Christoph Syldatk and Jens Rudat
}

\begin{abstract}
The amidase activities of two Aminobacter sp. strains (DSM24754 and DSM24755) towards the aryl-substituted substrates phenylhydantoin, indolylmethyl hydantoin, D,L-6-phenyl-5,6-dihydrouracil (PheDU) and para-chloro-D,L-6-phenyl-5,6-dihydrouracil were compared. Both strains showed hydantoinase and dihydropyrimidinase activity by hydrolyzing all substrates to the corresponding $N$-carbamoyl-a- or $\mathrm{N}$-carbamoyl- $\beta$-amino acids. However, carbamoylase activity and thus a further degradation of these products to $a$ - and $\beta$-amino acids was not detected. Additionally, the genes coding for a dihydropyrimidinase and a carbamoylase of Aminobacter sp. DSM24754 were elucidated. For Aminobacter sp. DSM24755 a dihydropyrimidinase gene flanked by two genes coding for putative ABC transporter proteins was detected. The deduced amino acid sequences of both dihydropyrimidinases are highly similar to the well-studied dihydropyrimidinase of Sinorhizobium meliloti CECT4114. The latter enzyme is reported to accept substituted hydantoins and dihydropyrimidines as substrates. The deduced amino acid sequence of the carbamoylase gene shows a high similarity to the very thermostable enzyme of Pseudomonas sp. KNK003A.
\end{abstract}

Keywords: Beta-amino acid, Dihydropyrimidinase, Hydantoinase, Carbamoylase

\section{Introduction}

Hydantoinases (EC 3.5.2.2) were thought to be the microbial counterparts of eukaryotic dihydropyrimidinases. For this reason the terms hydantoinase and dihydropyrimidinase are used synonymously in EC nomenclature. The eukaryotic enzymes catalyze the second step in the reductive pyrimidine degradation pathway by hydrolyzing the dihydropyrimidines dihydrouracil and dihydrothymine to the corresponding $N$-carbamoyl- $\beta$-amino acids (Vogels and van der Drift 1976). However, several hydantoinases are reported to lack the ability of hydrolyzing these natural substrates, e.g. D-hydantoinase from Bacillus thermocatenulatus GH-2, phenylhydantoinase from Escherichia coli and hydantoinase from Agrobacterium sp. IP 1-671 (Kim et al. 2000; Park et al. 1999; Runser and Meyer 1993). Therefore the natural function of hydantoinases is still unclear.

\footnotetext{
* Correspondence: ulrike.bretschneider@kit.edu

Karlsruhe Institute of Technology (KIT), Institute of Process Engineering in Life Sciences: Section II: Technical Biology, Engler-Bunte-Ring 1, 76131 Karlsruhe,
} Germany
Apart from that, hydantoinases are of high interest as they are utilized for the biocatalytic production of unnatural enantiopure $\alpha$-amino acids. In the so called hydantoinase process a racemic hydantoin is converted to a D- or L- $N$-carbamoyl- $\alpha$-amino acid and subsequently to a chiral D- or L- $\alpha$-amino acid applying a hydantoin racemase, a D- or L-specific hydantoinase and finally a D- or L-specific $N$-carbamoylase (see Figure $1 \mathrm{~A}$ ). This industrially applied process has a theoretical yield of $100 \%$. Nowadays there is also a rising demand for optically pure $\beta$-amino acids. These compounds are promising building blocks for pharmaceuticals and fine chemicals (Seebach and Gardiner 2008). However, the efficient production of chiral $\beta$-amino acids is still a challenging task (Weiner et al. 2010).

In a previous study we tested the potential of using a modified hydantoinase process for the production of optically pure $\beta$-amino acids (see Figure 1B). We demonstrated that three recombinant D-hydantoinases were able to convert aryl-substituted dihydropyrimidines to the corresponding $N$-carbamoyl- $\beta$-amino acids. Additionally, we detected

\section{Springer}




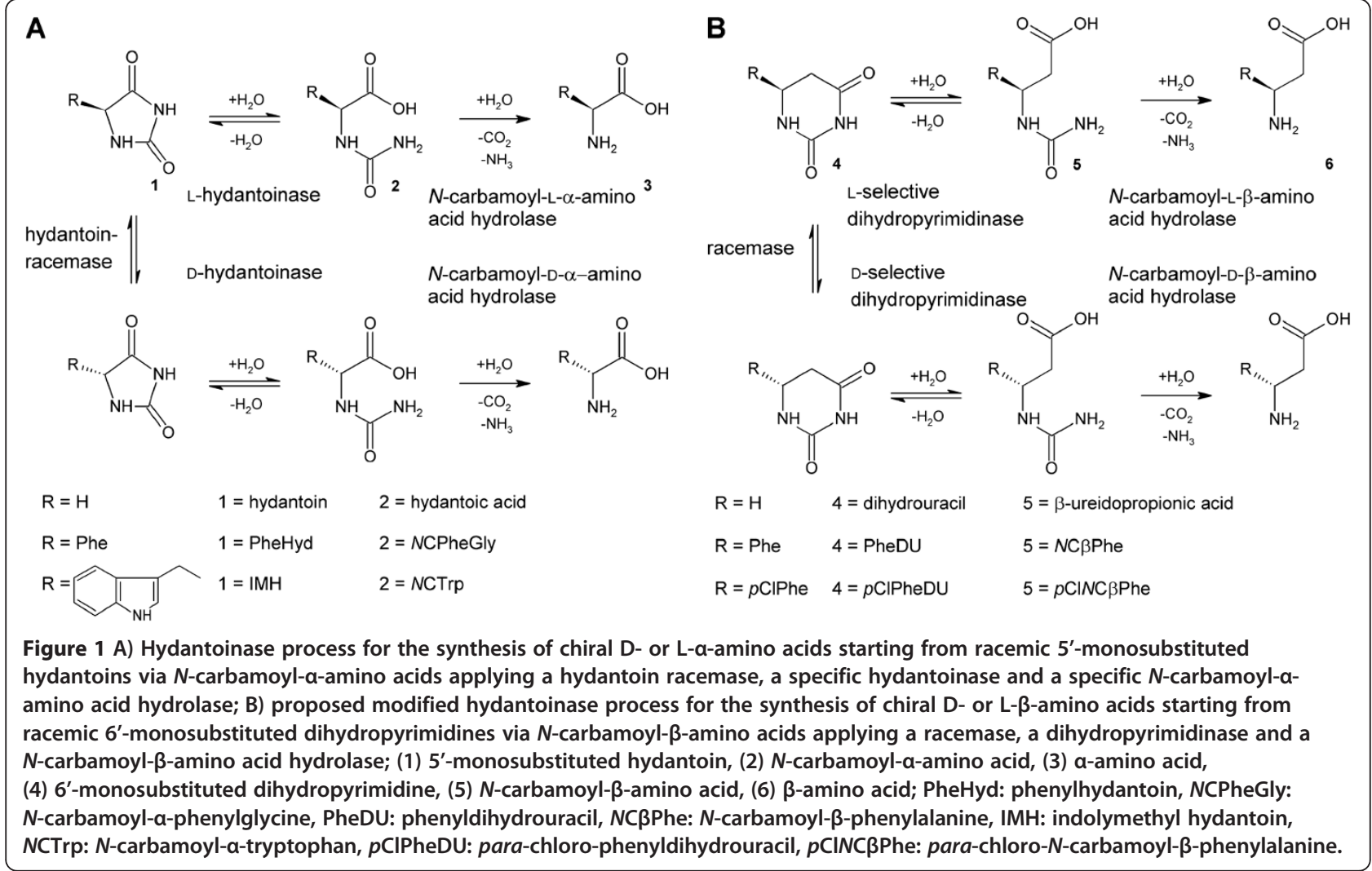

several bacterial strains exhibiting activity towards arylsubstituted dihydropyrimidines. Several bacterial isolates, among them Aminobacter sp. DSM24755, showed an enantioselective conversion of phenyldihydrouracil (Engel et al. 2011).

Here we report the dihydropyrimidinase and hydantoinase activities of two Aminobacter strains. Moreover the genes coding for a carbamoylase and a dihydropyrimidinase for Aminobacter sp. DSM24754 and a gene coding for another dihydropyrimidinase for Aminobacter sp. DSM24755 are described.

\section{Materials and methods}

\section{Chemicals}

Chemicals were of reagent grade and obtained from commercial sources if not stated otherwise. D,L-5-Indolylmethyl- $N$-3-methyl hydantoin $\left(\mathrm{CH}_{3}-\mathrm{IMH}\right)$ was supplied by former Degussa AG (now Evonik Industries AG). D- $\beta$ Phenylalanine (D- $\beta$ Phe) and L- $\beta$-phenylalanine (L- $\beta$ Phe) were obtained from Pep-Tech Corporation (Burlington, USA).

para-Chloro-D,L-phenyldihydrouracil ( $p$ ClPheDU) was provided from Fraunhofer Institute for Chemical Technology (Pfinztal Germany). Phenylhydantoin (PheHyd), 5 -indolylmethyl hydantoin (IMH) and $N$-carbamoyl- $\alpha$-tryptophan (NCTrp) were synthesized according to Stark and Smyth 1963; and Suzuki et al. 1973. Phenyldihydrouracil
(PheDU), N-rac-carbamoyl- $\beta$-phenylalanine (NC $\beta$ Phe) and L-NC $\beta$ Phe as standard for HPLC analysis were prepared according to Dakin and Dudley 1914 and Dürr 2007. The purity was proven by HPLC and ${ }^{1} \mathrm{H}-\mathrm{NMR}$. PheDU used for biotransformation experiments and para-chloro-D,L- $N$-carbamoyl- $\beta$-phenylalanine ( $p$ CINC $\beta$ Phe) were prepared as described elsewhere (Engel et al. 2011).

\section{Media}

The following media were used: Lysogeny broth (LB) medium modified after Bertani 1951 was used for cultivation of strains for biotransformation assays and growing cells for DNA extraction: $10 \mathrm{~g} / \mathrm{L}$ Bacto-tryptone, $5 \mathrm{~g} / \mathrm{L}$ yeast extract and $5 \mathrm{~g} / \mathrm{L} \mathrm{NaCl}$. The $\mathrm{pH}$ was adjusted to 7.2 with $\mathrm{NaOH}$. For $\mathrm{LBi}$ medium, $0.1 \mathrm{~g} / \mathrm{L} \mathrm{CH}_{3}-\mathrm{IMH}$ was added to LB.

\section{Bacterial strains}

The strains Aminobacter sp. DSM24754 and Aminobacter sp. DSM24755 were kindly provided by Dr. A. Puñal and first described by Engel et al. 2011.

\section{Cultivation conditions}

A bacterial colony was inoculated in $4 \mathrm{~mL} \mathrm{LB}$ at $30^{\circ} \mathrm{C}$ with $140 \mathrm{rpm}$ overnight. The resulting preculture was added to $100 \mathrm{~mL} \mathrm{LB}$ in a $1 \mathrm{~L}$ shaking flask and incubated at $30^{\circ} \mathrm{C}$ and $140 \mathrm{rpm}$. 


\section{Assay of enzyme activity}

Cells were harvested in the late exponential growth phase by centrifugation $\left(8000 \mathrm{x} \mathrm{g}, 10 \mathrm{~min}, 12^{\circ} \mathrm{C}\right)$. The supernatant was discarded and the cells were washed twice with potassium phosphate buffer $(0.1 \mathrm{M}, \mathrm{pH}$ 8) followed by centrifugation. Finally resting cells were obtained by resuspending the cells in a small volume of the same buffer.

Preparation of substrate solutions: The assay substrates PheDU, PheHyd and IMH were dissolved in potassium phosphate buffer $(0.1 \mathrm{M}, \quad \mathrm{pH} \quad 8)$ to a concentration of $4 \mathrm{mM}$ assisted by heating to $70^{\circ} \mathrm{C}$ for 30 min. $p$ ClPheDU was dissolved in DMSO to a concentration of $400 \mathrm{mM}$ due to its poor water solubility. $N C \beta P h e$ and NCTrp were dissolved to a concentration of $4 \mathrm{mM}$ in potassium phosphate buffer $(0.1 \mathrm{M}, \mathrm{pH} 8)$.

Biotransformation reactions with PheDU, IMH, PheHyd, $N C T r p$ or $N C \beta$ Phe were started by the addition of $500 \mu \mathrm{L}$ substrate solution to $500 \mu \mathrm{L}$ suspension of resting cells. For biotransformation reactions with $p$ ClPheDU $5 \mu \mathrm{L}$ of this substrate solution were added to $495 \mu \mathrm{L}$ of potassium phosphate buffer (0.1 M, pH 8) and subsequently biocatalysis was started by adding $500 \mu \mathrm{L}$ suspension of resting cells. All assays were carried out in a thermomixer at $40^{\circ} \mathrm{C}$, $1400 \mathrm{rpm}$ for $24 \mathrm{~h}$. Reactions were stopped by centrifugation $(13.000 \mathrm{x} \mathrm{g}, 1 \mathrm{~min})$. Supernatants were harvested and stored at $-28^{\circ} \mathrm{C}$ until analysis.

\section{Analysis}

All substrate and product concentrations were analyzed by HPLC on an Agilent 1100 system (Agilent Technologies, Santa Clara, USA) using a Nucleodur 100-5 C18 ec column (Macherey-Nagel, Germany). The mobile phase for the analysis of PheDU, NC $\beta$ Phe, $\beta$ Phe, IMH, NCTrp, tryptophan (Trp), PheHyd, $N$-carbamoyl- $\alpha$-phenylglycine (NCPheGly) and phenylglycine (PheGly) consisted of $20 \% \mathrm{MeOH} / 80 \%\left(0.1 \%(\mathrm{v} / \mathrm{v}) \mathrm{H}_{3} \mathrm{PO}_{4}\right.$ pH $\left.3.0(\mathrm{NaOH})\right)$. The flow rate was $0.8 \mathrm{~mL} / \mathrm{min}$, the temperature $30^{\circ} \mathrm{C}$ and the detection wavelength $210 \mathrm{~nm}$. For analysis of $p$ ClPheDU and $p$ CINC $\beta$ Phe the mobile phase was composed of $40 \% \mathrm{MeOH} / 60 \% 0.04 \mathrm{M}$ potassium phosphate buffer $\mathrm{pH}$ 6.5. The flow rate was $0.8 \mathrm{~min}$, the temperature $30^{\circ} \mathrm{C}$ and the detection wavelength $210 \mathrm{~nm}$.

\section{DNA preparation and sequencing of amidase genes}

To extract genomic DNA from bacterial isolates the ZR Soil Microbe DNA $\mathrm{Kit}^{\mathrm{TM}}$ was applied according to the manufacturer's instructions. Quality and quantity of genomic DNA were controlled by agarose gel electrophoresis using $1 \%$ agarose in TBE buffer. The gel was stained with ethidium bromide solution and analyzed under UV light.

Elucidation of amidase gene sequences is based on dihydropyrimidinase gene fragments amplified and provided by Dr. A. Puñal (Karlsruhe Institute of Technology
(KIT) former University of Karlsruhe) with the primers dhyd-f (AAACGGTT) (5'-GCCGCAGCATGCGGNGGN ACNAC-3') and dhyd-r (DADIVIWDPNGE) (5'-CACCA TTAGGGTCCCATATGACTADRTCNGCRT-3') for the strain Aminobacter sp. DSM24754 and the degenerate primers dhp-f (AAAFGG) (5'-GCSGCVTTYGGNGGNACN AC-3') and dhp-r (VHAENG) (5'-TCNCCRTTYTCNG CRTGNAC-3') for the strain Aminobacter sp. DSM24755 (Dürr 2007; Lin et al. 2005). The complete gene sequences were amplified by performing a modified TAIL-PCR. For the first TAIL-PCR the arbitrary degenerate primers AD1 and AD2 according to Liu and Whittier 1995 and the specific primers 728Tf1 (5'-GCTGCATATGTGCGTCAA TGGCTGG-3') and 728Tf2 (5'-GAGCGTGGCGTCAAC ACCTTCAAG-3') for strain Aminobacter sp. DSM24754 and the specific primers 735Tf1 (5'-GTCGACAAGG GCATCACCTCGTTC-3') and 735Tf2 (5'-GGTGGACGA CGACGAGATGTATTCG-3') for strain Aminobacter sp. DSM24755, synthesized by MWG-Biotech (Germany), were used. In contrast to the originally described method PCR products were separated after the secondary PCR on a $1 \%$ agarose gel. PCR products were purified with MinElute Gel Extraction Kit (Qiagen, Hilden Germany), ligated into pDrive PCR cloning vector (Qiagen, Hilden Germany) and sequenced (GATC Biotech AG, Germany). Subsequently the sequences were assembled with the known fragments. With the newly identified sequences further specific primers were designed and TAIL-PCR was conducted as described above until the gene sequences were elucidated completely. Sequence fragments were aligned using BioEdit program, BLAST was applied for comparison with other sequences. ClustalW2 was used for global sequence alignments to compare the new Aminobacter sequences with sequences in the NCBI database (Altschul et al. 1997; Altschul et al. 1990; Chenna et al. 2003). The aligned sequences were printed with ESPript (Gouet et al. 1999). For comparison of the Aminobacter sp. DSM24754 gene cluster with other prokaryotic genomes the program Comparative Genome Cluster Viewer (CGCV, Revanna et al. 2009) was applied.

\section{Results}

\section{Biotransformation experiments}

Due to previous results Aminobacter sp.DSM24754 was cultivated in LB and strain Aminobacter sp. DSM24755 in LBi for all experiments (Engel et al. 2011). The dihydropyrimidinase, hydantoinase and carbamoylase activities of Aminobacter sp. DSM24754 were tested. The results are summarized in Figure 2. Besides dihydropyrimidinase activity towards PheDU and $p$ ClPheDU hydantoinase activity towards PheHyd and IMH was detected for both strains. Under the chosen conditions PheDU and PheHyd were the best substrates for Aminobacter sp. DSM24754 and PheHyd 

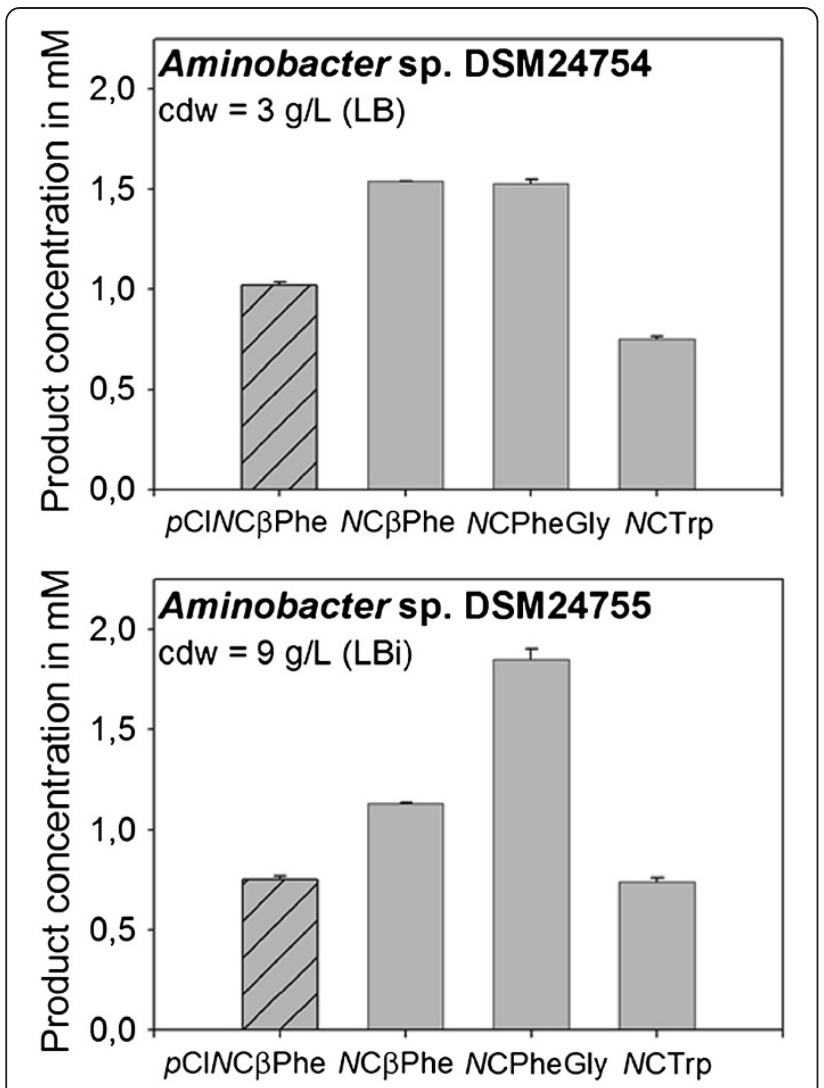

\section{$\mathrm{N}$-Carbamoyl amino acid concentration after incubation for $24 \mathrm{~h}$ with: \\ $2 \mathrm{mM}$ substrate \\ $2 \mathrm{mM}$ substrate in $0.5 \%$ DMSO}

Figure 2 Product concentrations measured after $24 \mathrm{~h}$ of resting cell biotransformation experiments with Aminobacter sp. DSM24754 compared to the results determined for Aminobacter sp. DSM24755 with four different substrates: para-Chloro-phenyldihydrouracil ( $\mathrm{pClPheDU}$ ) is converted to para-chloro- $N$-carbamoyl- $\beta$-phenylalanine ( $p$ CINC $\beta P h e)$, phenyldihydrouracil (PheDU) is converted to $N$-carbamoyl- $\beta$ phenylalanine (NCBPhe); phenylhydantoin (PheHyd) is converted to $N$-carbamoyl-a-phenylglycine (NCPheGly) and indolymethyl hydantoin (IMH) is converted to $\mathrm{N}$-carbamoyl-atryptophan (NCTrp). The substrate concentrations were $2 \mathrm{mM}$; $c d w=$ cell dry weight.

was the best substrate for Aminobacter sp. DSM24755. None of the Aminobacter strains exhibited carbamoylase activity towards the $N$-carbamoyl- $\beta$-amino acid $N C \beta P h e$ or the $N$-carbamoyl- $\alpha$-amino acid NCTrp.

\section{Identification of amidase genes}

A modified TAIL-PCR was conducted for both strains based on dihydropyrimidinase gene fragments. With this method a 3252 base pairs (bp) long DNA fragment from strain Aminobacter sp. DSM24754 and a 3127 bp long DNA fragment from Aminobacter sp. DSM24755 were amplified and subsequently sequenced.

\section{Sequence analysis of the genomic DNA fragment from Aminobacter sp. DSM24754}

The 3252 bp genomic DNA fragment of Aminobacter sp. DSM24754 has an overall GC content of 62\% and comprises two complete open reading frames (ORFs) and one incomplete ORF all starting with an ATG start codon (see Table 1).

The first ORF consists of 942 bp coding for a protein of 313 amino acids. It has the highest amino acid identity of $85 \%$ to the primary sequence of nonputative $N$-carbamoyl-D-amino acid amidohydrolase of Pseudomonas sp. KNK003A (BAD00008.1). ORF2 is located downstream to ORF1 and points into the same direction. It is either $1485 \mathrm{bp}$ or $1458 \mathrm{bp}$ long, as there is a second ATG start codon 27 bp downstream to the first. The real start codon has to be determined by a heterologous expression of the longer and the shorter version of the gene and subsequent activity tests. However, most of the similar enzymes in the database are aligning beginning with the second start codon. A possible Shine-Dalgarno sequence (RBS) is only detected upstream the second ATG codon (see Table 1). For these reasons all following identity data are related to this shorter gene product coding for a protein of 485 amino acids. This protein shares highest amino acid identity (83\%) with a putative dihydropyrimidinase of Mesorhizobium sp. BNC1 (YP_675206). The third ORF is $326 \mathrm{bp}$ long, incomplete and points into the opposite direction. Its deduced amino acid sequence shows highest identity of $78 \%$ to a hypothetical protein of Agrobacterium radiobacter K84 (YP_002542208.1). The possible function of the gene product of ORF3 remains unclear as it exhibits no clear similarity to non-putative proteins.

The sequence of the $3252 \mathrm{bp}$ genomic DNA fragment of Aminobacter sp. DSM24754 was deposited at EMBL database [HE651322].

\section{Sequence analysis of the genomic DNA fragment from Aminobacter sp. DSM24755}

The 3127 bp DNA fragment has an overall GC content of $63 \%$ and harbors two complete ORFs and one incomplete ORF (see Table 1). All ORFs point into the same direction and start with an ATG start codon.

The first ORF is $1452 \mathrm{bp}$ long and encodes a protein of 483 amino acids. This protein shows highest amino acid identity (90\%) to the putative dihydropyrimidinase of Mesorhizobium loti MAFF303099 (NP_103173.1). A second ORF of $822 \mathrm{bp}$ is located downstream to ORF1. Its deduced amino acid sequence displays highest identity (91\%) to a putative ATP-binding protein of an 
Table 1 Location and properties of genes and deduced proteins of the sequenced fragments of Aminobacter sp. DSM24754 and Aminobacter sp. DSM24755 respectively

\begin{tabular}{|c|c|c|c|c|c|c|c|c|c|c|}
\hline Aminobacter sp. & Gene & $\begin{array}{l}\text { Nucleotide } \\
\text { position }\end{array}$ & GC (\%) & Putative RBS & $\begin{array}{l}\text { Protein } \\
\text { length } \\
\text { (aa) }\end{array}$ & $\begin{array}{l}\text { Calculated } \\
\text { molecular } \\
\text { mass (kDa) }\end{array}$ & $\begin{array}{l}\text { Protein with highest } \\
\text { identity in BLAST }\end{array}$ & $\begin{array}{l}\text { Accession } \\
\text { number }\end{array}$ & Organism & $\begin{array}{c}\text { Identical } \\
\text { amino } \\
\text { acids (\%) }\end{array}$ \\
\hline \multirow[t]{4}{*}{ DSM24754 } & ORF1 & $274-1215$ & 62 & TGAGGAGGTAAGACCATG & 313 & 35 & $\begin{array}{l}\text { N-carbamoyl-D-amino } \\
\text { acid amidohydrolase }\end{array}$ & BAD00008.1 & Pseudomonas sp. KNK003A & 85 \\
\hline & \multirow[t]{2}{*}{ ORF2 } & $(1238) /$ & $(62.5) /$ & \multirow{2}{*}{$\begin{array}{l}\text { ATGACGGCAATCAAT } \\
\text { GGGAGAACGAGCATG }\end{array}$} & $(494) /$ & $(53.8) /$ & \multirow[t]{2}{*}{ putative dihydropyrimidinase } & \multirow[t]{2}{*}{ YP_675206 } & \multirow[t]{2}{*}{ Mesorhizobium sp. BNC1 } & \multirow[t]{2}{*}{83} \\
\hline & & $1265-2722$ & 62.7 & & 485 & 52.9 & & & & \\
\hline & ORF3 & $3252-2818$ & 59.8 & - & - & - & hypothetical protein & YP_002542208 & Agrobacterium radiobacter K84 & 78 \\
\hline \multirow[t]{3}{*}{ DSM24755 } & ORF1 & 80-1531 & 63.6 & TAGGGGAACTGAAGAAATG & 483 & 53.2 & putative phenylhydantoinase & NP_103173.1 & Mesorhizobium loti MAFF303099 & 90 \\
\hline & ORF2 & $1780-2601$ & 62.8 & TGGGGTAAAACCGGGCAATG & 273 & 67.5 & $\begin{array}{l}\text { putative ATP-binding } \\
\text { protein of ABC transporter }\end{array}$ & NP_103169.1 & Mesorhizobium loti MAFF303099 & 91 \\
\hline & ORF3 & $2607-3127$ & 62.8 & - & - & - & $\begin{array}{l}\text { putative permease protein } \\
\text { of } A B C \text { transporter }\end{array}$ & ZP_09089625.1 & $\begin{array}{l}\text { Mesorhizobium amorphae } \\
\text { CCNWGS0123 }\end{array}$ & 88 \\
\hline
\end{tabular}

$(-)$ no data available, due to an incomplete open reading frame. GC=guanine-cytosine content; aa =amino acids, $\left(^{*}\right)=$ values in brackets are for the 1485 bp long gene fragment of ORF 2 ; underlined sequence parts = putative Shine-Dalgarno sequence (RBS), sequence in boldface $=$ assumed start codon, sequence in italics = potential alternative start codon. 
ABC transporter of Mesorhizobium loti MAFF303099 (NP_103169.1). The incomplete third ORF starts only 4 bp downstream to ORF2 and consists of $521 \mathrm{bp}$. The deduced partial amino acid sequence exhibits highest identity $(88 \%)$ to the transmembrane component of an $\mathrm{ABC}$ transporter of Mesorhizobium amorphae CCNWGS0123 (ZP_09089625.1).

The 3127 bp genomic DNA fragment of Aminobacter sp. DSM24755 was deposited at EMBL database [HE651323].

The two genes coding for putative dihydropyrimidinases of the Aminobacter sp. strains DSM24754 and DSM24755 have an overall gene sequence identity of $74 \%$. The identity of the deduced amino acid sequences is $68 \%$.

\section{Discussion}

\section{Biotransformation results}

In this study the dihydropyrimidine, hydantoin and carbamoyl amino acid hydrolyzing abilities of Aminobacter sp. DSM24754 were determined. Both Aminobacter strains showed dihydropyrimidinase and hydantoinase activity towards PheDU, $p$ ClPheDU, PheHyd and IMH by converting these substrates to the corresponding $N$-carbamoyl $\beta$ - and $N$-carbamoyl- $\alpha$-amino acids. Aminobacter sp. DSM24754 hydrolyzed the same amount of PheHyd and PheDU under the chosen conditions. Aminobacter sp. DSM24755 also showed a high conversion of PheHyd but in contrast to the results obtained for the other Aminobacter strain the dihydropyrimidine PheDU was not hydrolyzed to the same extend. This may be due to the fact that a D-selectivity for PheDU is described for Aminobacter sp. DSM24755 while Aminobacter sp. DSM24754 is reported to be unselective for this substrate (Engel et al. 2011). Nothing is known about the stereoselectivity for PheHyd of both biocatalysts. But this substrate is known to spontaneously racemize under alkaline conditions because of its keto-enoltautomerism (Pietzsch and Syldatk 1995). For this reason a potential stereoselectivity of the biocatalyst would probably not influence the biotransformation reaction. The dihydropyrimidine PheDU cannot racemize spontaneously due to its different chemical structure. Thus a stereoselective biocatalyst would hydrolyze this substrate less effective.

When only the hydantoins PheHyd and IMH were compared PheHyd appeared to be the better substrate for both biocatalysts as a higher amount of this compound was degraded, respectively. This hydantoin is composed of a phenyl group directly bound to the hydantoin ring, which is highly similar to PheDU consisting of a phenyl group linked to a dihydropyrimidine ring. By contrast, the bulky aromatic indol ring of IMH is connected to the hydantoin ring via a methyl bridge. This difference in substrate structure may be a reason for the differences observed in our experiments. In general D-hydantoinases preferably hydrolyze

phenyl-substituted hydantoins while L-hydantoinases show higher activities towards benzyl-substituted hydantoins such as IMH which is assumed to be a consequence of their different three-dimensional structures (Abendroth et al. 2002a).

A further degradation of the resulting $N$-carbamoyl amino acids was not observed. Additionally, no carbamoylase activities were detectable in resting cell biotransformation experiments directly applying NCTrp and NC $\beta P$ Phe as substrates. We see two possible explanations for these results: Either (i) there are no genes coding for carbamoylases present in the genomes of the two tested Aminobacter strains or (ii) the experimental settings prevented the detection of carbamoylase activity. For example the expression of carbamoylases is reported to be inducible (Mei et al. 2008). Consequently the lack of an inducer during growth of Aminobacter sp. DSM24754 or the use of the wrong inducer $\left(\mathrm{CH}_{3}-\mathrm{IMH}\right)$ during growth of Aminobacter sp. DSM24755 may account for not detectable carbamoylase activities. Otherwise it may be that carbamoylases were expressed but not active with the substrates tested. Explanation (ii) seems to be more plausible as at least for strain Aminobacter sp. DSM24754 a gene product coding for a D-carbamoylase was detected (see above).

\begin{tabular}{|c|c|c|c|c|c|}
\hline \multirow{2}{*}{$\begin{array}{l}\text { Amino acid sequence identities and similarities } \\
\text { of carbamoylases }\end{array}$} & DSM24754 & BAD00008.1 & 1FO6 & 1ERZ & ABS11194.1 \\
\hline & \multicolumn{5}{|c|}{ Identity \% } \\
\hline Aminobacter sp. DSM24754 & & 85 & 58 & 57 & 49 \\
\hline Pseudomonas sp. KNK003 BAD00008.1 & 92 & & 59 & 58 & 51 \\
\hline Agrobacterium radiobacter CCRC14924 $\mathbf{1 F O 6}$ & 68 & 70 & & 96 & 57 \\
\hline A. sp. KNK712 1ERZ & 69 & 70 & 97 & & 56 \\
\hline A. tumefaciens RU-OR HyuC2 ABS11194.1 & 56 & 57 & 64 & 93 & \\
\hline
\end{tabular}




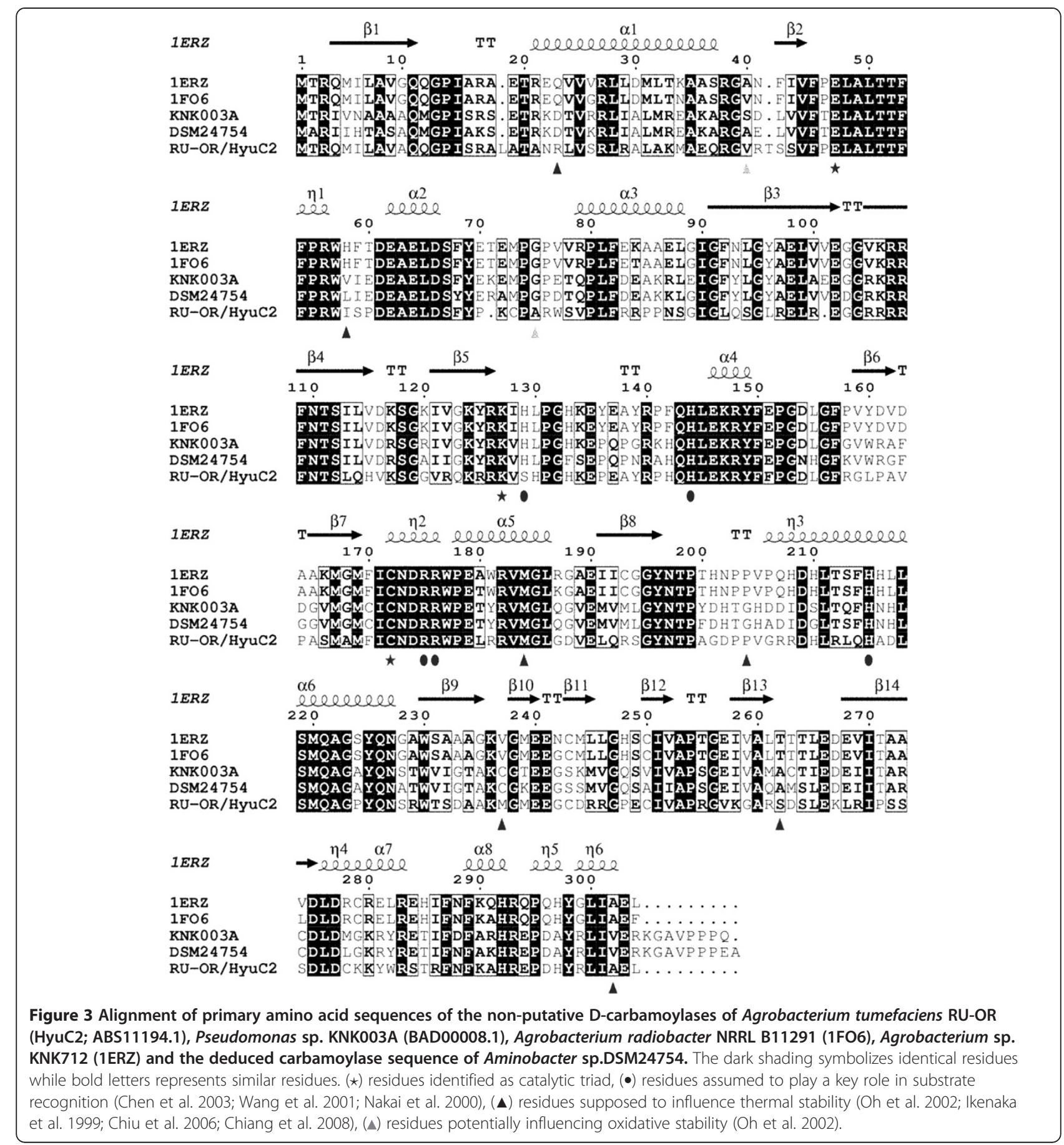

Comparison with other functionally related carbamoylases

For strain Aminobacter sp. DSM24754 a gene coding for a carbamoylase was identified and the deduced amino acid sequence showed highest amino acid identity (85\%) to the D-carbamoylase of Pseudomonas sp. KNK003A (BAD00008.1, Ikenaka et al. 1998). Furthermore it exhibited $49-58 \%$ identity to three well studied Dcarbamoylases of Agrobacterium radiobacter CCRC14924
(1FO6) Agrobacterium sp. KNK712 (1ERZ) and Agrobacterium tumefaciens RU-OR (HyuC2; ABS11194.1) within a global alignment (see Table 2 and Figure 3; Wang et al. 2001; Nakai et al. 2000; Jiwaji et al. 2009).

In this alignment important residues were annotated and the residue numbers refer to the protein sequence of Aminobacter sp. DSM24754. All of the aligned nonputative enzymes are D-carbamoylases able to catalyze the hydrolytic cleavage of $N$-carbamoyl- $\alpha$-amino acids. 
For none of these enzymes activity towards $N$-carbamoyl$\beta$-amino acids is described (Martínez-Rodríguez. et al. 2010a). The residues identified as the catalytic triad (Glu47, Lys127, Cys172) and the residues assumed to play a key role in substrate recognition (His129, His144, Arg175, Arg176, His215) are highly conserved for all sequences aligned (Nakai et al. 2000; Wang et al. 2001; Chen et al. 2003). Due to its sequence characteristics the Aminobacter sp. DSM24754 enzyme can also be classified as D-carbamoylase.

The putative carbamoylase of Aminobacter sp. DSM24754 exhibited highest identity to the Pseudomonas sp. KNK003A D-carbamoylase, which is described to be the most thermostable carbamoylase known today (Martínez-Rodríguez et al. 2010a). However, the low stability of D-carbamoylases is one major drawback for their efficient use in an industrial hydantoinase process. Therefore various attempts have been made to engineer their thermal and oxidative stability. For the carbamoylase of $A$. radiobacter CCRC14924 it was reported that the residues Gln23, His58, Met184, Val237 and Thr262 influence the stability against temperature and oxidation, the residues Val40 and Gly75 affect the oxidative stability solely (Oh et al. 2002; Chiang et al. 2008), and that the residue Ala302 influences thermostability and catalytic activity (Chiu et al. 2006). Thermal and $\mathrm{pH}$ stability of the carbamoylase of Agrobacterium sp. KNK712 was shown to be affected by the residues His58, Pro204 and Val237 (Ikenaka et al. 1999). Except for Met184, all residues described to be involved in thermal stability of the two Agrobacterium enzymes differ from the residues in the carbamoylase sequences of Aminobacter sp. DSM24754 and Pseudomonas sp. KNK003A. Remarkably 5 of these 6 substitutions (Asp23, Gly204, Cys237, Ala262, Val302) are identical while one is similar (Val/Leu58) for the both latter enzymes. Furthermore in the D-carbamoylase of $A$. radiobacter CCRC14924 a single mutation of Thr262 to
Ala led to a significant increase in oxidative and thermal stability (Oh et al. 2002). The enzymes of Pseudomonas sp. KNK003A and Aminobacter sp. DSM24754 already possess an Ala residue in this position. Due to its sequences characteristics the Aminobacter sp. DSM24754 carbamoylase could have a similar high temperature stability like the Pseudomonas sp. KNK003A D-carbamoylase. This is to be studied in more detail within further experiments by its recombinant expression and biochemical characterization.

The recently discovered second carbamoylase of $A$. tumefaciens RU-OR (HyuC2) has as well substitutions in four positions (Arg24, Ile60, Met237, Ser262) compared to the above mentioned seven residues probably important for thermal stability (Jiwaji et al. 2009). Thus the authors assumed that the thermal and oxidative stability of the enzyme may differ from those of the other well characterized Agrobacterium enzymes. However, the exchanged residues are neither identical with the residues of Aminobacter sp. DSM24754 carbamoylase nor with the corresponding residues of the Pseudomonas sp. KNK003A carbamoylase protein sequence.

\section{Comparison with other functionally related hydantoinases} Within a BLAST search against the protein database the deduced amino acid sequence of DSM24754 ORF2 exhibited highest identity (83\%) to a putative dihydropyrimidinase of Mesorhizobium sp. BNC1. Surprisingly the second BLAST hit was with the non-putative hydantoinase of Pseudomonas sp. KNK003A (BAE20330.1) having an overall identity of $82 \%$. This is the same Pseudomonas strain whose carbamoylase showed highest identity to Aminobacter sp. DSM24754 carbamoylase (see section above). When compared to other non-putative enzymes (see Table 3 and Figure 4) the identities were $70-73 \%$ to the Ochrobactrum sp. G21 D-hydantoinase (ABS84244.1), to the D-hydantoinase of Jannaschia sp. CCS1 (YP_510647.1)

\begin{tabular}{|c|c|c|c|c|c|c|c|}
\hline \multirow{2}{*}{$\begin{array}{l}\text { Amino acid sequence identities and similarities } \\
\text { of hydantoinases }\end{array}$} & DSM24754 & DSM24755 & BAE20330.1 & ABS84244.1 & YP_510647.1 & $3 \mathrm{DC} 8$ & K1D \\
\hline & \multicolumn{7}{|c|}{ Identity \% } \\
\hline Aminobacter sp. DSM24754 & & 69 & 82 & 73 & 73 & 70 & 42 \\
\hline Aminobacter sp. DSM24755 & 83 & & 68 & 74 & 68 & 79 & 46 \\
\hline Pseudomonas sp. KNK003A BAE20330.1 & 91 & 82 & & 73 & 71 & 69 & 42 \\
\hline Ochrobactrum sp. G21 ABS84244.1 & 86 & 87 & 86 & & 69 & 71 & 42 \\
\hline Jannaschia sp. CCS1 YP_510647.1 & 85 & 80 & 83 & 81 & & 68 & 42 \\
\hline Sinorhizobium meliloti CECT4114 3 DC8 & 81 & 89 & 81 & 84 & 78 & & 44 \\
\hline \multirow[t]{2}{*}{ Bacillus stearothermophilus SD1 1K1D } & 59 & 64 & 61 & 60 & 59 & 61 & \\
\hline & \multicolumn{7}{|c|}{ Similarity \% } \\
\hline
\end{tabular}




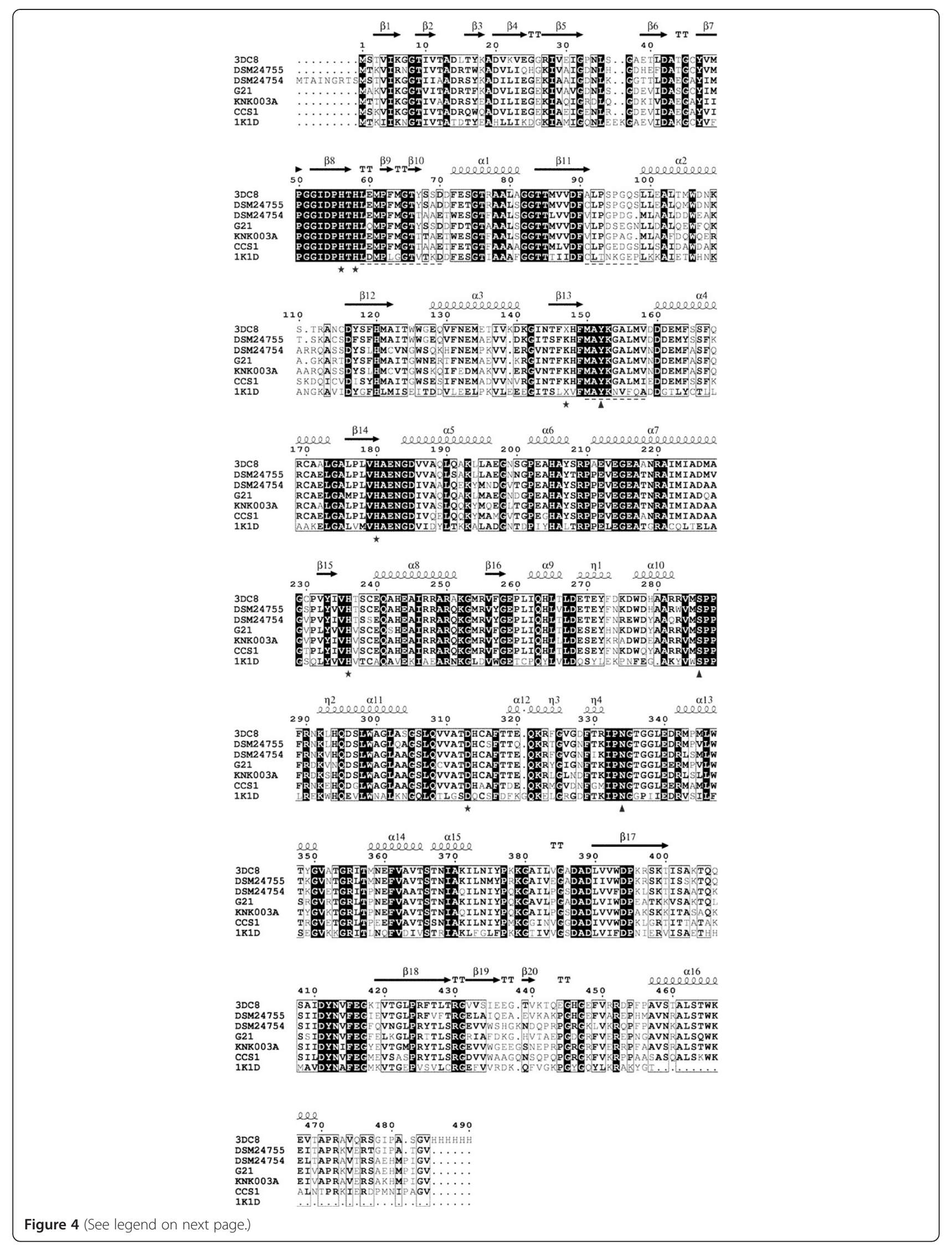


(See figure on previous page.)

Figure 4 Alignment of primary amino acid sequences of the non-putative dihydropyrimidinase of Sinorhizobium meliloti CECT4114 (3DC8), D-hydantoinase of Ochrobactrum sp. G21 (ABS84244.1), D-hydantoinase of Pseudomonas sp. KNK003A (BAE20330.1), Jannaschia sp. CCS1 (YP_510647.1) and D-hydantoinase of Bacillus stearothermophilus SD1 (1K1D) and the newly identified dihydropyrimidinase sequences of Aminobacter sp. DSM24754 and Aminobacter sp. DSM24755. The dark shading symbolizes identical residues while bold letters represents similar residues. (-) stereochemistry gate loops (SGL1: 59-70; SGL2: 91-98, SGL3: 150-158), (*) residues assumed to build the catalytic core, $(\mathbf{\Lambda})$ residues assumed to be involved in the recognition the hydantoin ring (Martínez-Rodríguez et al. 2010a).

and to the dihydropyrimidinase of Sinorhizobium meliloti CECT4114 (3 DC8) (Dürr et al. 2008; Cai et al. 2009; Martinez-Rodriguez et al. 2010b). The identity to the wellstudied D-hydantoinase of Bacillus stearothermophilus SD1 (1K1D) was $42 \%$ (Cheon et al. 2002). The overall identity to the deduced dihydropyrimidinase of Aminobacter sp. DSM24755 was $68 \%$ only, although both gene products were detected in strains belonging to the same genus.

The deduced dihydropyrimidinase of DSM24755 ORF1 showed highest identity of $91 \%$ to the putative phenylhydantoinase of Mesorhizobium loti MAFF303099 in a BLAST search. The highest overall identity of $79 \%$ to a nonputative protein was detected with the dihydropyrimidinase of S. meliloti CECT4114. A global alignment with further non-putative enzymes (see Table 3 and Figure 4) showed that the amino acid identities to Ochrobactrum sp. G21 Dhydantoinase, to the D-hydantoinase of Pseudomonas sp. KNK003A and to the D-hydantoinase of Jannaschia sp. CCS1 were $74 \%$ to $68 \%$. The primary amino acid identity to the D-hydantoinase of the B. stearothermophilus SD1 was only $46 \%$.

In the global alignment of the protein sequences the residues described to be important for structure or function were annotated (see Figure 4). The following residue numbers refer to the deduced amino acid sequence of Aminobacter sp. DSM24755 dihydropyrimidinase. Like other dihydropyrimidinases the deduced Aminobacter sp. DSM24754 and Aminobacter sp. DSM24755 enzymes possess the highly conserved GxxDxHxH motif (residues 51-58) (May et al. 1998). Together with the histidin residues of this motif the residues Lys146, His179, His 235 and Asp312 are suggested to form the catalytic core and are as well completely conserved within the aligned proteins. Furthermore the lysine residue (Lys146) is post-translationally carboxylated in most known hydantoinases/dihydropyrimidinases (Abendroth et al. 2002b; Cheon et al. 2002). It is confirmed for several hydantoinases/dihydropyrimidinases that these active site residues form a binuclear center with divalent metal ions (MartínezRodríguez et al. 2010b; Zhang et al. 2010). Due to the fact that these residues are completely conserved in the protein sequences of Aminobacter sp. DSM24754 and Aminobacter sp. DSM24755 and due to the overall high sequence similarity with the well-studied dihydropyrimidinase of S. meliloti CECT4114 a metal dependence of these newly described enzymes is most likely.
The C-termini of hydantoinases are supposed to be involved in quaternary structure composition (Kim and Kim 1998, 2002). This region was described to be non-homologous among microbial hydantoinases. However, it was recently reported that the $\mathrm{C}$-termini of hydantoinases are highly conserved for $\alpha$-Proteobacteria (MartinezRodriguez et al. 2010b). The C-terminal regions of the deduced amino acid sequences of the $\alpha$-Proteobacteria strains Aminobacter sp. DSM24754 and Aminobacter sp. DSM24755 support this assumption. They are highly homologous to the C-termini of S. meliloti CECT4114 dihydropyrimidinase, Ochrobactrum sp. G21 D-hydantoinase, and Jannaschia sp. CCS1 hydantoinase (see Figure 4).

For S. meliloti CECT4114 it is assumed that the substrate's hydantoin ring is recognized by residues Tyr152, Ser286 and Asn334, which are highly conserved among hydantoinases (Martínez-Rodríguez et al. 2010b). The corresponding residues in Aminobacter sp. DSM24754 and Aminobacter sp. DSM24755 dihydropyrimidinases are identical. The exocyclic side chain of the substrate is reported to be recognized by the so called stereochemistry gate loops (SGL), which are less conserved. It was suggested that these SGLs may be involved in determining the substrate specificity of these enzymes (Cheon et al. 2002). For the two Aminobacter sp. dihydropyrimidinases the residues of SGL3 (see Figure 4) are identical to each other and to S. meliloti CECT4114 dihydropyrimidinase, Ochrobactrum sp. G21 hydantoinase and Pseudomonas sp. KNK003A hydantoinase and are highly similar to the Jannaschia sp. CCS1 hydantoinase. There are bigger differences with regard to SGL1 and SGL2 among the two Aminobacter enzymes. The Aminobacter sp. DSM24754 dihydropyrimidinase SGL1 and SGL2 residues are nearly identical to residues of SGL1 and SGL2 in Jannaschia sp. CCS1 hydantoinase. In contrast the SGL1 and SGL2 residues of Aminobacter sp. DSM24755 dihydropyrimidinase almost completely match with the residues of S. meliloti CECT4114 dihydropyrimidinase. These results could indicate that the substrate specificity of the two Aminobacter sp. dihydropyrimidinases may be slightly different. This would correspond to the results obtained in the resting cell biotransformation experiments with the two strains.

In resting cell biocatalysis experiments Aminobacter sp. DSM24754 and Aminobacter sp. DSM24755 exhibited hydantoinase and dihydropyrimidinase activity. This raises 


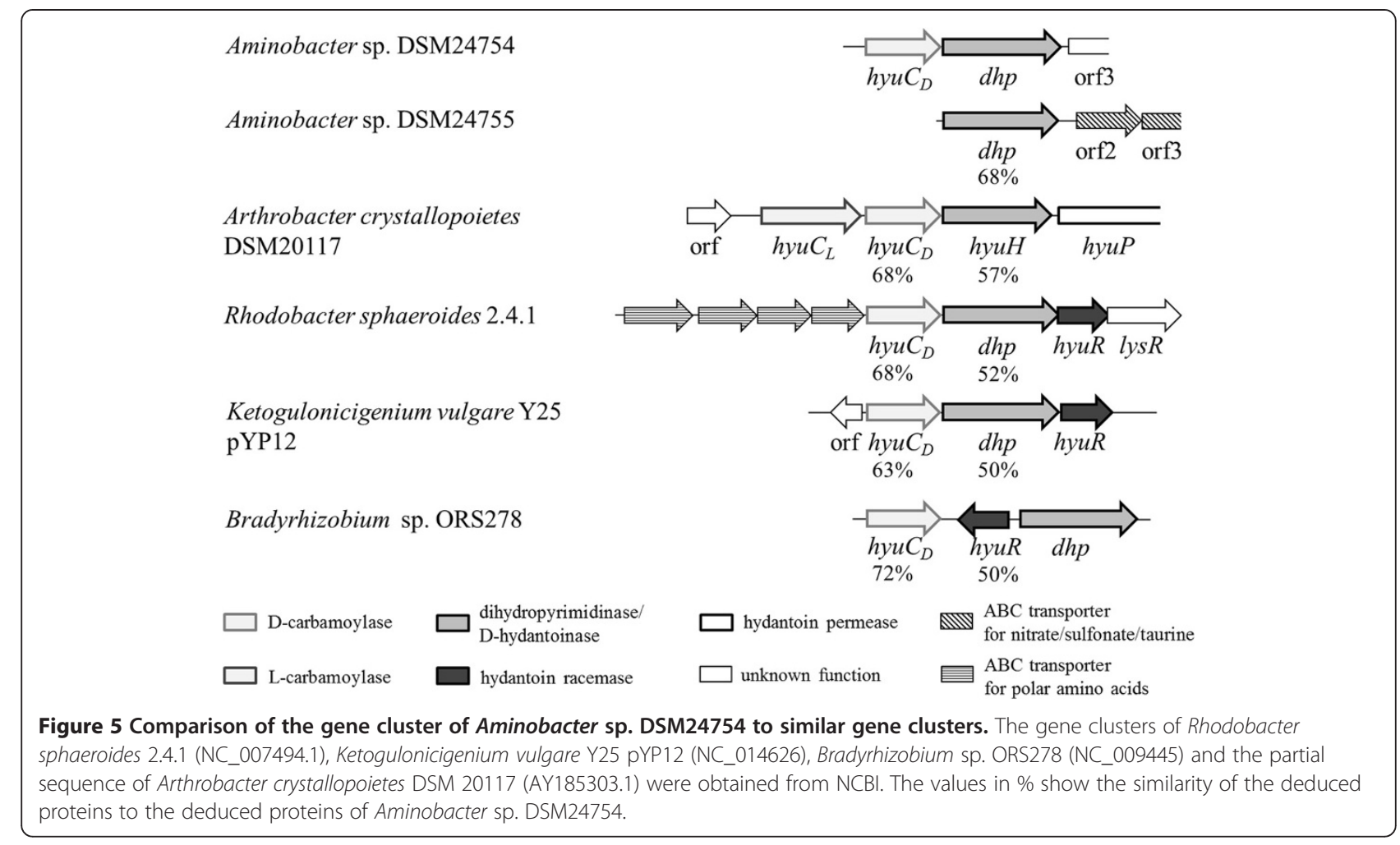

the question whether these dihydropyrimidinase and the hydantoinase activities originate from one enzyme or two different enzymes, a hydantoinase and a dihydropyrimidinase in the respective strain. The $S$. meliloti CECT4114 dihydropyrimidinase is described to hydrolyze substituted five-membered and also six-membered ring substrates (Martínez-Rodríguez et al. 2010b). In a previous study we reported that Ochrobactrum sp. G21 hydantoinase, Delftia sp. I24 hydantoinase and Arthrobacter crystallopoietes DSM20117 hydantoinase can act on substituted hydantoins and on aryl-substituted dihydropyrimidines (Engel et al. 2011). The hydantoinase of Jannaschia sp. CCS1 is described to accept hydantoins but showed highest activity towards dihydrouracil (Cai et al. 2009). However, nothing is reported concerning the substrate specificity of Pseudomonas sp. KNK003A hydantoinase (Ikenaka et al. 1998). Due to the fact that most of the similar hydantoinases/dihydropyrimidinases have hydantoinase and dihydropyrimidinase activity we hypothesize that the detected deduced dihydropyrimidinases of Aminobacter sp. DSM24754 and Aminobacter sp. DSM24755 are responsible for both measured activities as well. The major difference was that strain Aminobacter sp. DSM24755 showed a D-stereoselectivity for phenyldihydrouracil while strain Aminobacter sp. DSM24754 did not (Engel et al. 2011). Whether this is related to the differences in the primary structure of the dihydropyrimidinases has to be elucidated in further experiments with the pure enzymes.

\section{Comparison to other hydantoin/dihydropyrimidine cleaving gene clusters}

Bacterial hydantoin utilizing $(h y u)$ genes especially hydantoinases/dihydropyrimidinases and carbamoylases are often organized in gene clusters (Dürr et al. 2008). Compared to other described hyu gene clusters the arrangement of the D-carbamoylase $(h y u C)$ gene upstream to the D-hydantoinase $(h y u H)$ gene in Aminobacter sp. DSM24754 appears to be unusual. To our knowledge Arthrobacter crystallopoietes DSM20117 is the only bacterial strain with a similar gene organization and approved hydantoinase and carbamoylase activities reported in literature (Werner et al. 2004). The similarity of the two Aminobacter sp. dihydropyrimidinases to the D-hydantoinase protein of A. crystallopoietes DSM20117 is $57 \%$ and the similarity of Aminobacter sp. DSM24754 carbamoylase to the $\mathrm{D}$-carbamoylase protein of $A$. crystallopoietes DSM20117 is 68\%.

A database search against prokaryotic genomes resulted in only eight hits showing a comparable gene organization of putative hyuC and hyuH genes (see Figure 5). In the chromosome of four Rhodobacter strains ( $R$. sphaeroides ATCC 17025, R. sphaeroides ATCC 17029, R. sphaeroides 2.4.1, R. sphaeroides KD131) and on the plasmid pYP12 of Ketogulonicigenium vulgare Y25 the same arrangement of the D-carbamoylase gene and the D-hydantoinase gene as observed in Aminobacter sp. DSM24754 was detected. A similar gene organization was found in three 
Bradyrhizobium strains with the difference that a gene coding for a hydantoin racemase pointing in the opposite direction (for Bradyrhizobium sp. BTAi1, Bradyrhizobium sp. ORS278) or two hypothetical genes (for B. japonicum USDA 110) are located between hyuC and hyuH.

The Rhodobacter sphaeroides strains and Ketogulonicigenium vulgare Y25 harbor two genes coding for hydantoinases respectively. The second hydantoinase forms a hyu gene cluster with a $\beta$-ureidopropionase ( $R$. sphaeroides) or an L-carbamoylase ( $K$. vulgare Y25) located upstream to the hydantoinase gene. The similarity of Aminobacter sp. DSM24754 and DSM24755 dihydropyrimidinases to the $R$. sphaeroides and $K$. vulgare Y25 hydantoinases clustering with the $\beta$-ureidopropionases or L-carbamoylase is higher (66-89\%) than to the hydantoinases clustering with the D-carbamoylases (50-53\%).

The Bradyrhizobium strains possess three genes coding for D-hydantoinases and harbor three to four genes coding for D-carbamoylases, respectively. However, only one hyu cluster composed of a D-carbamoylase and hydantoinase is found in each strain. The sequence similarity of the Aminobacter sp. dihydropyrimidinases to the Bradyrhizobium hydantoinases not forming a cluster with a D-carbamoylase is again higher (49-78\%) than to the hydantoinases clustering with a D-carbamoylase (47-51\%). The similarities of Bradyrhizobium D-carbamoylases clustering with a hydantoinase to the Aminobacter sp. carbamoylases are $71-74 \%$ while the similarities to the other carbamoylases are between 46 and $74 \%$.

This raises the questions whether or not the two Aminobacter sp. strains also possess several hydantoinases and carbamoylases and what role the hyu genes and their clustering play for these strains.

\section{Competing interests}

The authors declare that they have no competing interests.

\section{Acknowledgements}

This work was financed by the Federal Ministry of Science and Education (BMBF), Germany. Furthermore the authors like to thank Gerd Unkelbach from Fraunhofer Institute for Chemical Technology for the cooperation in the synthesis of the above mentioned substrates.

Received: 26 February 2012 Accepted: 15 May 2012

Published: 27 June 2012

\section{References}

Abendroth J, Niefind K, May O, Siemann M, Syldatk C, Schomburg D (2002a) The structure of L-hydantoinase from Arthrobacter aurescens leads to an understanding of dihydropyrimidinase substrate- and enantiospecificity. Biochemistry 41(27):8589-8597

Abendroth J, Niefind K, Schomburg D (2002b) X-ray structure of a dihydropyrimidinase from Thermus sp. at 1.3 angstrom resolution. J Mol Biol 320(1):143-156

Altschul SF, Gish W, Miller W, Myers EW, Lipman DJ (1990) Basic local alignment search tool. J Mol Biol 215(3):403-410

Altschul SF, Madden TL, Schaffer AA, Zhang JH, Zhang Z, Miller W, Lipman DJ (1997) Gapped BLAST and PSI-BLAST: a new generation of protein database search programs. Nucleic Acids Res 25(17):3389-3402

Bertani G (1951) Studies on Lysogenesis.1. The mode of phage liberation by lysogenic Escherichia coli. J Bacteriol 62(3):293-300
Cai Y, Trodler P, Jiang S, Zhang W, Wu Y, Lu Y, Yang S, Jiang W (2009) Isolation and molecular characterization of a novel. D-hydantoinase from Jannaschia sp. CCS1 FEBS J 276(13):3575-3588, doi:10.1111/j.1742-4658.2009.07077.x

Chen CY, Chiu WC, Liu JS, Hsu WH, Wang WC (2003) Structural basis for catalysis and substrate specificity of Agrobacterium radiobacter $\mathrm{N}$-carbamoyl-D-amino acid amidohydrolase. J Biol Chem 278(28):26194-26201

Chenna R, Sugawara H, Koike T, Lopez R, Gibson TJ, Higgins DG, Thompson JD (2003) Multiple sequence alignment with the Clustal series of programs. Nucleic Acids Res 31(13):3497-3500

Cheon YH, Kim HS, Han KH, Abendroth J, Niefind K, Schomburg D, Wang JM, Kim Y (2002) Crystal structure of D-hydantoinase from Bacillus stearothermophilus: Insight into the stereochemistry of enantioselectivity. Biochemistry 41 (30):9410-9417

Chiang CJ, Chern JT, Wang JY, Chao YP (2008) Facile immobilization of evolved Agrobacterium radiobacter carbamoylase with high thermal and oxidative stability. J Agric Food Chem 56(15):6348-6354

Chiu W-C, You J-Y, Liu J-S, Hsu S-K, Hsu W-H, Shih C-H, Hwang J-K, Wang W-C (2006) Structure-stability-activity relationship in covalently cross-linked $\mathrm{N}$ carbamoyl D-amino acid amidohydrolase and $\mathrm{N}$-acylamino acid racemase. J Mol Biol 359(3):741-753

Dakin HD, Dudley HW (1914) The resolution of inactive uramido-acids and hydantoins into active components, and their conversion into amino-acids. I. b-Phenyl-a-uramidopropionic acid, benzylhydantoin and phenylalanine. J Biol Chem 17(1):29-36

Dürr R (2007) Screening and description of novel hydantoinases from distinct environmental sources. Doctoral Thesis, Universität Karlsruhe (TH), Karlsruhe. ISBN 978-3-86644-173-6

Dürr R, Neumann A, Vielhauer O, Altenbuchner J, Burton SG, Cowan DA, Syldatk C (2008) Genes responsible for hydantoin degradation of a halophilic Ochrobactrum sp. G21 and Delftia sp. 124 - New insight into relation of Dhydantoinases and dihydropyrimidinases. Journal of Molecular Catalysis B: Enzymatic 52-3:2-12

Engel U, Syldatk C, Rudat J (2011) Stereoselective hydrolysis of aryl-substituted dihydropyrimidines by hydantoinases. Appl Microbiol Biotechnol. doi:DOI 10.1007/s00253-011-3691-7

Gouet P, Courcelle E, Stuart DI, Metoz F (1999) ESPript: analysis of multiple sequence alignments in PostScript. Bioinformatics 15(4):305-308

Ikenaka Y, Nanba H, Yamada Y, Yajima K, Takano M, Takahashi S (1998) Screening, characterization, and cloning of the gene for $\mathrm{N}$-carbamyl-D-amino acid amidohydrolase from thermotolerant soil bacteria. Biosci Biotechnol Biochem 62(5):882-886

Ikenaka Y, Nanba H, Yajima K, Yamada Y, Takano M, Takahashi S (1999) Thermostability reinforcement through a combination of thermostabilityrelated mutations of $\mathrm{N}$-carbamyl-D-amino acid amidohydrolase. Biosci Biotechnol Biochem 63(1):91-95

Jiwaji M, Hartley CJ, Clark SA, Burton SG, Dorrington RA (2009) Enhanced hydantoin-hydrolyzing enzyme activity in an Agrobacterium tumefaciens strain with two distinct $\mathrm{N}$-carbamoylases. Enzym Microb Technol 44(4):203-209. doi:DOI 10.1016/j.enzmictec.2008.11.006

Kim GJ, Kim HS (1998) C-terminal regions of D-hydantoinases are nonessential for catalysis, but affect the oligomeric structure. Biochem Biophys Res Commun 243(1):96-100

Kim GJ, Kim HS (2002) A microbial D-hydantoinase is stabilized and overexpressed as a catalytically active dimer by truncation and insertion of the C-terminal region. J Microbiol Biotechnol 12(2):242-248

Kim GJ, Lee DE, Kim HS (2000) Functional expression and characterization of the two cyclic amidohydrolase enzymes, allantoinase and a novel phenylhydantoinase, from Escherichia coli. J Bacteriol 182(24):7021-7028

Lin LL, Hsu WH, Hsu WY, Kan SC, Hu HY (2005) Phylogenetic analysis and biochemical characterization of a thermostable dihydropyrimidinase from alkaliphilic Bacillus sp. TS-23. Antonie Van Leeuwenhoek International Journal of General and. Mol Microbiol 88(3-4):189-197

Liu YG, Whittier RF (1995) Thermal Asymmetric Interlaced PCR - Automatable amplification and sequencing of insert end fragments from P1 and YAC clones for chromosome walking. Genomics 25(3):674-681

Martínez-Rodríguez S, Martinez-Gomez A, Rodriguez-Vico F, Clemente-Jimenez J, Las Heras-Vazquez FJ (2010a) Carbamoylases: characteristics and applications in biotechnological processes. Appl Microbiol Biotechnol 85 (3):441-458. doi:10.1007/s00253-009-2250-y

Martínez-Rodríguez S, Martinez-Gomez Al, Clemente-Jimenez JM, Rodriguez-Vico F, Garcia-Ruiz JM, Las Heras-Vazquez FJ, Gavira JA (2010b) Structure of 
dihydropyrimidinase from Sinorhizobium meliloti CECT4114: New features in an amidohydrolase family member. J Struct Biol 169(2):200-208. doi:10.1016/ j.jsb.2009.10.013

May O, Habenicht A, Mattes R, Syldatk C, Siemann M (1998) Molecular evolution of hydantoinases. Biol Chem 379(6):743-747

Mei Y, He B, Ouyang P (2008) Enzymatic production of L-amino acids from the corresponding D,L-5-substituted hydantoins by Bacillus fordii MH602. World J Microbiol Biotechnol 24(3):375-381, doi:10.1007/s11274-007-9485-5

Nakai T, Hasegawa T, Yamashita E, Yamamoto M, Kumasaka T, Ueki T, Nanba H, Ikenaka Y, Takahashi S, Sato M, Tsukihara T (2000) Crystal structure of Ncarbamyl-D-amino acid amidohydrolase with a novel catalytic framework common to amidohydrolases. Structure 8(7):729-738

Oh KH, Nam SH, Kim HS (2002) Improvement of oxidative and thermostability of $\mathrm{N}$-carbamyl-D-amino acid amidohydrolase by directed evolution. Protein Engineering 15(8):689-695

Park JH, Kim GJ, Lee SG, Lee DC, Kim HS (1999) Purification and characterization of thermostable D-hydantoinase from Bacillus thermocatenulatus GH-2. Appl Biochem Biotechnol 81(1):53-65

Pietzsch M, Syldatk C (1995) Hydrolysis and Formation of Hydantoins. Version. In: Drauz K, Waldmann H (eds) Enzyme Catalysis in Organic Synthesis. WileyVCH, Weinheim, pp 409-431. doi:10.1002/9783527618262.ch12d. ISBN 3527284796

Revanna KV, Krishnakumar V, Dong Q (2009) A web-based software system for dynamic gene cluster comparison across multiple genomes. Bioinformatics 25(7):956-957. doi:10.1093/bioinformatics/btp078

Runser SM, Meyer PC (1993) Purification and biochemical characterization of the hydantoin hydrolyzing enzyme from Agrobacterium species: A hydantoinase with no 5,6-dihydropyrimidine amidohydrolase activity. Eur J Biochem 213 (3):1315-1324

Seebach D, Gardiner J (2008) b-Peptidic peptidomimetics. Accounts of Chemical Research 41(10):1366-1375. doi:10.1021/ar700263g

Stark GR, Smyth DG (1963) Use of cyanate for determination of $\mathrm{NH}_{2}$-terminal residues in proteins. J Biol Chem 238(1):214-226

Suzuki T, Igarashi K, Hase K, Tuzimura K (1973) Optical-rotatory dispersion and circular-dichroism of amino-acid hydantoins. Agric Biol Chem 37(2):411-416

Vogels GD, van der Drift C (1976) Degradation of purines and pyrimidines by microorganisms. Bacteriol Rev 40(2):403-468

Wang W-C, Hsu W-H, Chien F-T, Chen C-Y (2001) Crystal structure and sitedirected mutagenesis studies of $\mathrm{N}$-carbamoyl-D-amino-acid amidohydrolase from Agrobacterium radiobacter reveals a homotetramer and insight into a catalytic cleft. J Mol Biol 306(2):251-261

Weiner B, Szymanski W, Janssen DB, Minnaard AJ, Feringa BL (2010) Recent advances in the catalytic asymmetric synthesis of b-amino acids. Chem Soc Rev 39(5):1656-1691

Werner M, Las Heras-Vazques FJ, Fritz C, Vielhauer O, Siemann-Herzberg M, Altenbuchner J, Syldatk C (2004) Cloning of D-specific hydantoin utilization genes from Arthrobacter crystallopoietes. Eng Life Sci 4(6):563-572

Zhang XY, Yuan JM, Niu LX, Liang AH (2010) Quantitative analysis and functiona evaluation of zinc ion in the D-hydantoinase from Pseudomonas putida YZ26. BioMetals 23(1):71-81

doi:10.1186/2191-0855-2-33

Cite this article as: Engel et al:: Novel amidases of two Aminobacter sp. strains: Biotransformation experiments and elucidation of gene sequences. AMB Express 2012 2:33.

\section{Submit your manuscript to a SpringerOpen ${ }^{\circ}$ journal and benefit from:}

- Convenient online submission

- Rigorous peer review

- Immediate publication on acceptance

- Open access: articles freely available online

- High visibility within the field

- Retaining the copyright to your article

Submit your next manuscript at $\gg$ springeropen.com 